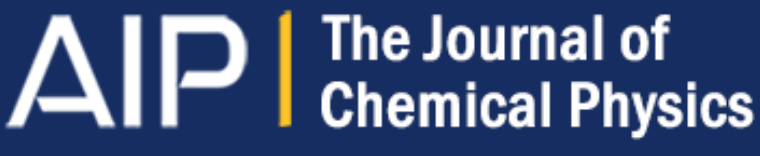

\section{Electron dynamics upon ionization: Control of the timescale through chemical substitution and effect of nuclear motion}

Morgane Vacher, David Mendive-Tapia, Michael J. Bearpark, and Michael A. Robb

Citation: The Journal of Chemical Physics 142, 094105 (2015); doi: 10.1063/1.4913515

View online: http://dx.doi.org/10.1063/1.4913515

View Table of Contents: http://scitation.aip.org/content/aip/journal/jcp/142/9?ver=pdfcov

Published by the AIP Publishing

\section{Articles you may be interested in}

Photoelectron spectroscopic study of the $\mathrm{E} \otimes \mathrm{e}$ Jahn-Teller effect in the presence of a tunable spin-orbit interaction. I. Photoionization dynamics of methyl iodide and rotational fine structure of $\mathrm{CH} 3 \mathrm{I}+$ and CD3I+ J. Chem. Phys. 134, 054308 (2011); 10.1063/1.3547548

Infrared spectroscopy of ionized corannulene in the gas phase

J. Chem. Phys. 134, 054310 (2011); 10.1063/1.3540661

Photodissociation and photoionization of 2,5-dihydroxybenzoic acid at 193 and $355 \mathrm{~nm}$

J. Chem. Phys. 133, 244309 (2010); 10.1063/1.3518709

Photoionization-induced dynamics of ammonia: Ab initio potential energy surfaces and time-dependent wave packet calculations for the ammonia cation

J. Chem. Phys. 124, 214306 (2006); 10.1063/1.2202316

The Jahn-Teller effect in the lower electronic states of benzene cation. III. The ground-state vibrations of C 6 $\mathrm{H} 6+$ and $\mathrm{C} 6 \mathrm{D} 6+$

J. Chem. Phys. 120, 8587 (2004); 10.1063/1.1691818

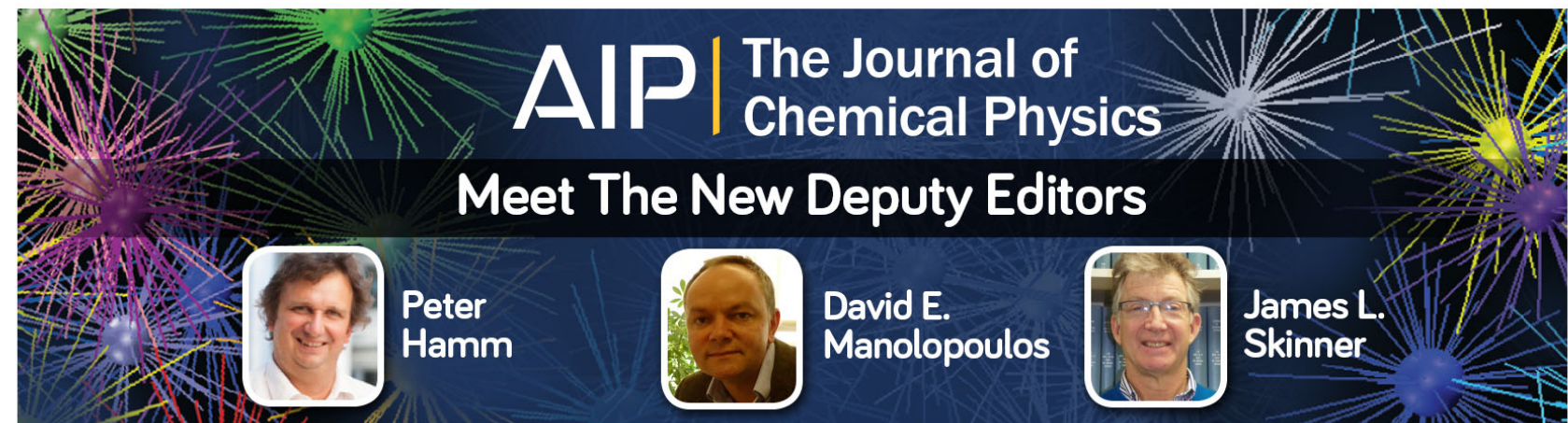




\title{
Electron dynamics upon ionization: Control of the timescale through chemical substitution and effect of nuclear motion
}

\author{
Morgane Vacher, ${ }^{1}$ David Mendive-Tapia, ${ }^{2}$ Michael J. Bearpark, ${ }^{1}$ and Michael A. Robb ${ }^{1}$ \\ ${ }^{1}$ Department of Chemistry, Imperial College London, London SW7 2AZ, United Kingdom \\ ${ }^{2}$ Laboratoire CEISAM - UMR CNR 6230, Université de Nantes, 2 Rue de la Houssinière, BP 92208, \\ 44322 Nantes Cedex 3, France
}

(Received 21 December 2014; accepted 12 February 2015; published online 2 March 2015)

\begin{abstract}
Photoionization can generate a non-stationary electronic state, which leads to coupled electronnuclear dynamics in molecules. In this article, we choose benzene cation as a prototype because vertical ionization of the neutral species leads to a Jahn-Teller degeneracy between ground and first excited states of the cation. Starting with equal populations of ground and first excited states, there is no electron dynamics in this case. However, if we add methyl substituents that break symmetry but do not radically alter the electronic structure, we see charge migration: oscillations in the spin density that we can correlate with particular localized electronic structures, with a period depending on the gap between the states initially populated. We have also investigated the effect of nuclear motion on electron dynamics using a complete active space self-consistent field (CASSCF) implementation of the Ehrenfest method, most previous theoretical studies of electron dynamics having been carried out with fixed nuclei. In toluene cation for instance, simulations where the nuclei are allowed to move show significant differences in the electron dynamics after $3 \mathrm{fs}$, compared to simulations with fixed nuclei. (C) 2015 AIP Publishing LLC. [http://dx.doi.org/10.1063/1.4913515]
\end{abstract}

\section{INTRODUCTION}

Photoionization can create a coherent superposition of electronic states and therefore initiate electron dynamics in atoms and molecules. The interference between electronic eigenstates in such a superposition, alternating between constructive and destructive, leads to oscillating motion of the electronic density with a period inversely proportional to the energy gap. ${ }^{1,2}$ This is "pure" electron dynamics (i.e., takes place even if the nuclei are fixed) and is often called charge migration in the literature.

Observing and controlling electron dynamics is a target of attosecond spectroscopy. ${ }^{3-7}$ Molecular experiments to investigate such dynamics are particularly difficult to interpret as changes in nuclear geometry are also expected while electron dynamics is occurring. The computational cost of a full quantum mechanical treatment of both electron and nuclear dynamics is high. Therefore, most electron dynamics simulations $^{8-13}$ are done with the fixed-nuclei approximation. The justification given for this is the difference in timescales of electron and nuclear dynamics, the electron distribution changing much faster than the nuclear geometry. A theoretical study by Nest and Ulusoy ${ }^{14}$ suggests that the effect of nuclear motion is very small during the first ten or so femtoseconds, as it takes time for the nuclei to move sufficiently to influence the electronic superposition. We have previously studied molecules ${ }^{15,16}$ where electron dynamics is affected by the nuclear motion after only a few fs and others ${ }^{15,17}$ when it seems to survive for several tens of fs. One of the purposes of this article is to understand further the extent to which neglecting nuclear motion is a reasonable approximation.
The other purpose of this work is to study the effect of chemical substitution on electron dynamics. Cederbaum et al. have simulated the process (still at fixed geometries) for three molecules differing in the initially ionized chromophore site but having nearly identical amine-acceptor sites: 2phenylethyl-N,N-dimethylamine (PENNA), 3-methylen-4penten-N,N-dimethylamine (MePeNNA), and 3-buten-N,Ndimethylamine (BUNNA). ${ }^{11}$ They showed that, as the nature of the eigenstates of the cation is molecule-dependent, the nature of the hole created and the magnitude of its migration will also be molecule-dependent. Also, ionization may result in electron dynamics with different timescales because the energy gap between the populated eigenstates varies.

In this article, we investigate the role of chemical substitution considering three molecules where the different substituent groups perturb only slightly the electronic structure and do not radically alter it. We choose benzene, and methylsubstituted benzenes - toluene and para-xylene-as examples because vertical ionization takes place at geometries near the conical intersections (CIs) between ground and first excited states of their cations.

Figure 1 represents the ionization process in benzene. The equilibrium geometry of the neutral species is exactly on the seam of CI. A projection of the surrounding "moat" of the conical intersection ${ }^{18-20}$ is shown. It contains two types of valence bond (VB) resonance structures: three quinoid (which are minima on the ground state surface) and three antiquinoid (which are transition structures on the ground state surface). In toluene and para-xylene, the equilibrium geometry of the neutral species is slightly displaced from the CI in their cation, resulting in an energy gap between the cationic ground and first excited states at this geometry. 


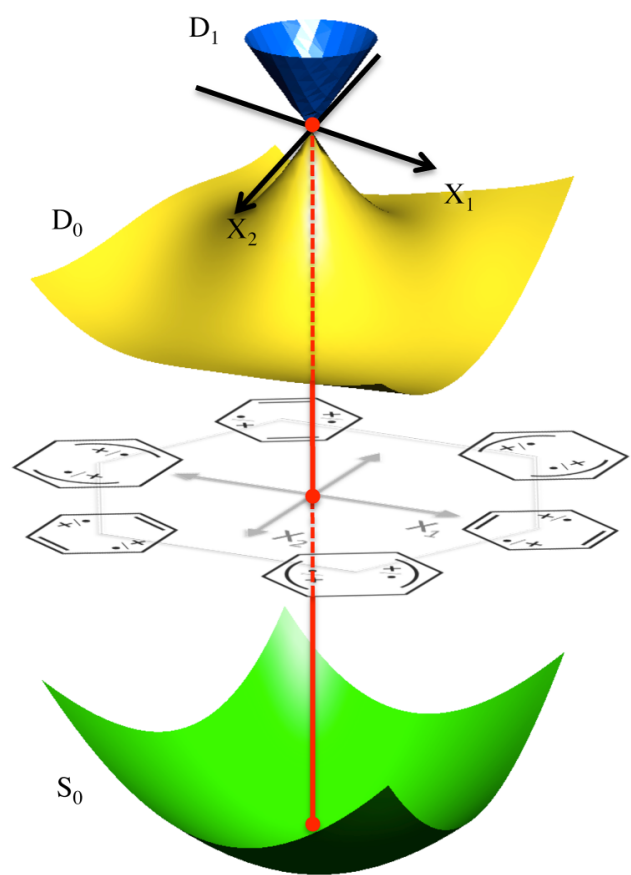

FIG. 1. The ionization process in benzene: the lower surface represents the ground state of the neutral species and the upper two represent the ground and first excited states of the cation in the branching space of the conical intersection $\left(X_{1}\right.$ is the gradient difference vector and $X_{2}$ the derivative coupling vector). A projection of the cationic conical intersection moat expressed in term of valence bond structures is shown centrally. Note that for each quinoid or antiquinoid structure, there are actually two resonance structures with the unpaired electron and the positive charge exchanged (as indicated by $\bullet /+$ ).

CIs are geometries where two electronic states (at least) are degenerate. They are important in photochemistry as they allow efficient non-radiative electronic transitions ${ }^{21-23}$ and may lead to several products. As the energy gap decreases, the electron dynamics slows down to the femtosecond timescale, which is the timescale of nuclear motion. ${ }^{24}$ The justification for the fixed-nuclei approximation-different timescales for electron and nuclear dynamics - therefore does not hold in regions of the potential energy surfaces near CIs. ${ }^{25}$

Using our complete active space self-consistent field (CASSCF) implementation of the Ehrenfest method, ${ }^{16}$ we can study the evolution of a non-stationary electronic wavefunction for fixed nuclei, and where the nuclei are allowed to move, to investigate the differences. ${ }^{15,17}$ We can therefore study the influence of the nuclear motion on the electron dynamics and see to what extent the fixed-nuclei approximation is valid. Note that in this work, we focus on the electron dynamics affected by the nuclear motion, not on the nuclear motion (studied in detail in our previous article ${ }^{26}$ ).

\section{THEORETICAL METHOD}

We have simulated the coupled electron-nuclear dynamics using our CASSCF implementation of the Ehrenfest method. ${ }^{16}$ Here, we only provide a summary and illustrate the method with a two-state system (as we will consider the two lowestenergy states populated in the molecules studied).

The dynamics of a molecular system is given by the time-dependent Schrödinger equation, where $r$ and $R$ refer to the electronic and nuclear variables, respectively,

$$
i \hbar \frac{\partial}{\partial t} \Phi(r, R, t)=\mathcal{H} \Phi(r, R, t) .
$$

The following approximate ansatz is used for the total wavefunction:

$$
\Phi(r, R, t)=\Psi(r, t) \cdot \chi(R, t) .
$$

\section{A. Quantum electron dynamics}

Integrating Eq. (1) using product ansatz (2) and taking the classical limit for the nuclei gives the following equation for the quantum mechanical treatment of the electron dynamics: ${ }^{16}$

$$
i \hbar \frac{\partial \Psi(r, t ; R(t))}{\partial t}=\mathcal{H}_{e}(r ; R(t)) \cdot \Psi(r, t ; R(t)),
$$

where the electronic wavefunction $\Psi$ and the electronic Hamiltonian $\mathcal{H}_{e}$ depend parametrically on the nuclear positions $R(t)$. Integration of Eq. (3) gives

$$
\begin{gathered}
\Psi(r, t ; R(t))=\exp \left(-\frac{i}{\hbar} \int_{t=0}^{t} \mathcal{H}_{e}\left(r ; R\left(t^{\prime}\right)\right) \partial t^{\prime}\right) \\
. \Psi(r, t=0 ; R(t=0)) .
\end{gathered}
$$

In practice, time is discretized and the electronic Hamiltonian is assumed to be constant over the time step. The electronic wavefunction at the time step $t_{n}$ is therefore calculated from the wavefunction at the step $t_{n-1}$ using the following relation:

$$
\begin{aligned}
\Psi\left(r, t_{n} ; R\left(t_{n}\right)\right)= & \exp \left(-\frac{i}{\hbar} \mathcal{H}_{e}\left(r ; R\left(t_{n}\right)\right) \cdot\left(t_{n}-t_{n-1}\right)\right) \\
& \cdot \Psi\left(r, t_{n-1} ; R\left(t_{n-1}\right)\right) .
\end{aligned}
$$

We can expand the time-dependent electronic wavefunction in the basis of configurations: $\mathbf{A}\left(t_{n}\right)$ is the vector gathering the expansion coefficients at time $t_{n}$. We use bold font to signify vectors and matrices,

$$
\mathbf{A}\left(t_{n}\right)=\left(\begin{array}{c}
a_{1}\left(t_{n}\right) \\
\vdots \\
a_{k}\left(t_{n}\right) \\
\vdots
\end{array}\right) .
$$

Using the spectral resolution of the electronic Hamiltonian, Eq. (5) reads as

$$
\begin{aligned}
\mathbf{A}\left(t_{n}\right)= & \mathbf{U}\left(R\left(t_{n}\right)\right) \cdot \exp \left(-\frac{i}{\hbar} \mathbf{D}_{e}\left(R\left(t_{n}\right)\right) \cdot\left(t_{n}-t_{n-1}\right)\right) \\
& \cdot \mathbf{U}^{\dagger}\left(R\left(t_{n}\right)\right) \cdot \mathbf{A}\left(t_{n-1}\right),
\end{aligned}
$$

$\mathbf{U}$ is the matrix containing the CASSCF eigenvectors arranged as columns. $\mathbf{D}_{e}$ is the matrix representation of the electronic Hamiltonian in the basis of eigenstates: it thus contains the CASSCF eigenvalues $\left\{E_{k}\right\}$ on the diagonal and is zero elsewhere.

The sequence of time-dependent vectors $\{\mathbf{A}\}$ may be obtained keeping the nuclei fixed or in concert with nuclear motion by updating the nuclear geometry $R\left(t_{n}\right)$ at each time step. In the first case, pure electron dynamics is simulated, and in the latter, coupled electron-nuclear dynamics 
is simulated. Note that only the configuration expansion coefficients $\left\{a_{k}\left(t_{n}\right)\right\}$ are propagated; the molecular orbitals are re-optimised if the nuclei are allowed to move but they are assumed to change very little.

\section{B. Classical nuclear dynamics}

The nuclear dynamics is included, if wanted, using the approximate Ehrenfest method. The nuclear motion is treated classically by solving Newton's equation of motion

$$
\frac{d P_{I}}{d t}=-\nabla_{I}\left\langle\Psi(r, t)\left|\mathcal{H}_{e}(r ; R(t))\right| \Psi(r, t)\right\rangle_{r},
$$

with $P_{I}$ the classical momentum of nucleus $I$. We integrate Eq. (8) using the Hessian-based predictor-corrector algorithm designed by Hase and Schlegel ${ }^{27}$ in the Gaussian program. ${ }^{28}$ The first and second derivatives of the energy (computed using the methods of Almlöf and Taylor ${ }^{29}$ ) are used to make a local quadratic approximation of the energy. In addition, a $5^{\text {th }}$ order polynomial fit is used to correct the second-order method. The approximations made are detailed in our previous article. ${ }^{16}$

One limitation of the Ehrenfest method is the lack of electronic decoherence: the populated electronic eigenstates share the same nuclear geometry because of the ansatz for the total wavefunction (Eq. (2)). This could lead to non-physical asymptotic behaviors. It is not expected to be a problem here as we are interested in relatively short timescale dynamics. However, the validity of the approximation will require further testing in the future. Nest demonstrated that the effect of the nuclear motion on the electron dynamics is underestimated with mean-field approaches. ${ }^{14}$ One therefore expects larger effects when the nuclei can move in different directions on different potential energy surfaces. Ways to go beyond the approximation we have used would be to work with an exact factorization of the total wavefunction ${ }^{30-32}$ or to use a Hellertype Gaussian wavepacket representation ${ }^{33-35}$ of the nuclear wavepacket.

\section{Example of an electronic superposition of two states}

Let us consider a molecular system in a superposition of two adiabatic electronic states $\psi_{0}$ and $\psi_{1}$ with eigenvalues $E_{0}$ and $E_{1}$, respectively. Initially, the electronic wavefunction reads as

$$
\Psi(r, t=0 ; R)=c_{0} \cdot \psi_{0}(r ; R)+c_{1} \cdot \psi_{1}(r ; R),
$$

where $c_{0}$ and $c_{1}$ are the (complex) expansion coefficients in the adiabatic basis. The normalization of the electronic superposition is ensured by $\left|c_{0}\right|^{2}+\left|c_{1}\right|^{2}=1$.

\section{With fixed nuclei}

If the nuclei are kept fixed, the electronic Hamiltonian becomes time-independent. Equation (4) reads then exactly as

$$
\Psi(r, t ; R)=\exp \left(-\frac{i}{\hbar} \mathcal{H}_{e}(r ; R) \cdot t\right) \cdot \Psi(r, t=0 ; R),
$$

where the nuclear geometry $R$ is now time-independent. Expanding the electronic wavefunction in the adiabatic basis, we obtain

$$
\begin{aligned}
\Psi(r, t ; R)= & c_{0} \exp \left(-\frac{i}{\hbar} E_{0}(R) \cdot t\right) \cdot \psi_{0}(r ; R) \\
& +c_{1} \exp \left(-\frac{i}{\hbar} E_{1}(R) \cdot t\right) \cdot \psi_{1}(r ; R),
\end{aligned}
$$

where the eigenvalues $E_{0}(R)$ and $E_{1}(R)$ do not depend on time. Note that there is no transfer of population between the two adiabatic states. The corresponding probability density reads

$$
\begin{aligned}
|\Psi(r, t ; R)|^{2}= & \left|c_{0}\right|^{2}\left|\psi_{0}(r ; R)\right|^{2}+\left|c_{1}\right|^{2}\left|\psi_{1}(r ; R)\right|^{2} \\
& +2 \operatorname{Re}\left(c_{0}^{*} c_{1} \cdot \exp \left(i \frac{E_{0}(R)-E_{1}(R)}{\hbar} t\right)\right. \\
& \left.\cdot \psi_{0}^{*}(r ; R) \psi_{1}(r ; R)\right) .
\end{aligned}
$$

Assuming real wavefunctions and using $\phi=\arg \left(\frac{c_{1}}{c_{0}}\right)$ the relative phase between the two complex expansion coefficients, it then reads

$$
\begin{aligned}
|\Psi(r, t ; R)|^{2}= & \left|c_{0}\right|^{2}\left|\psi_{0}(r ; R)\right|^{2}+\left|c_{1}\right|^{2}\left|\psi_{1}(r ; R)\right|^{2} \\
& +2\left|c_{0}\right|\left|c_{1}\right| \cos \left(\frac{E_{0}(R)-E_{1}(R)}{\hbar} t+\phi\right) \\
& \cdot \psi_{0}(r ; R) \psi_{1}(r ; R) .
\end{aligned}
$$

The first two terms are the weighted probability densities of the two adiabatic states. The third term corresponds to interference between the two electronic states, alternating between constructive and destructive. The nature of the hole created and of the oscillating motion in the electronic density is determined by the electronic character of the adiabatic states and by their occupation via the first two terms and $\psi_{0}(r ; R) \psi_{1}(r ; R)$ in the third term of Eq. (13). The occupations of the two states also determine the amplitude of the oscillations via $\left|c_{0}\right|\left|c_{1}\right|$. The initial relative phase $\phi$ determines the oscillation amplitude at $t=0$; it can be seen as a shift of the time axis (in simulations with fixed nuclei). The period is inversely proportional to the energy gap between the two eigenstates,

$$
T=\frac{\mathrm{h}}{E_{0}(R)-E_{1}(R)} .
$$

\section{With nuclei moving}

When the nuclei are allowed to move, several quantities become time-dependent: (i) the electronic character of the adiabatic states $\psi_{0}(r ; R(t))$ and $\psi_{1}(r ; R(t))$, (ii) the energy gap $E_{0}(R(t))-E_{1}(R(t))$, and (iii) the magnitude of $c_{0}$ and $c_{1}$ because of population transfer (due to the non-adiabatic coupling). Equation (4) cannot be solved exactly in general and one has to approximate the integral by a sum over short time intervals where the electronic Hamiltonian is assumed to be constant (see Eq. (5)). One still expects oscillations in the electronic density but not "perfect" sinusoids, as electron and nuclear dynamics interact. 


\section{COMPUTATIONAL DETAILS}

\section{A. Electronic structure and nuclear trajectory}

The electronic structure is computed using the stateaveraged $\mathrm{CASSCF}^{36}$ method. Using the standard $6-31 \mathrm{G}^{*}$ basis set, we choose the $6 \pi$ orbitals as active. The nuclear dynamics is integrated using the Hessian-based predictorcorrector algorithm with a mass-weighted step size of $0.0025 \mathrm{amu}^{1 / 2}$ bohrs (corresponding to a time step of about $0.04 \mathrm{fs})$.

\section{B. Analysis tools}

As detailed in our previous work, ${ }^{16}$ we suggest using the diagram shown centrally in Figure 1 to represent both the
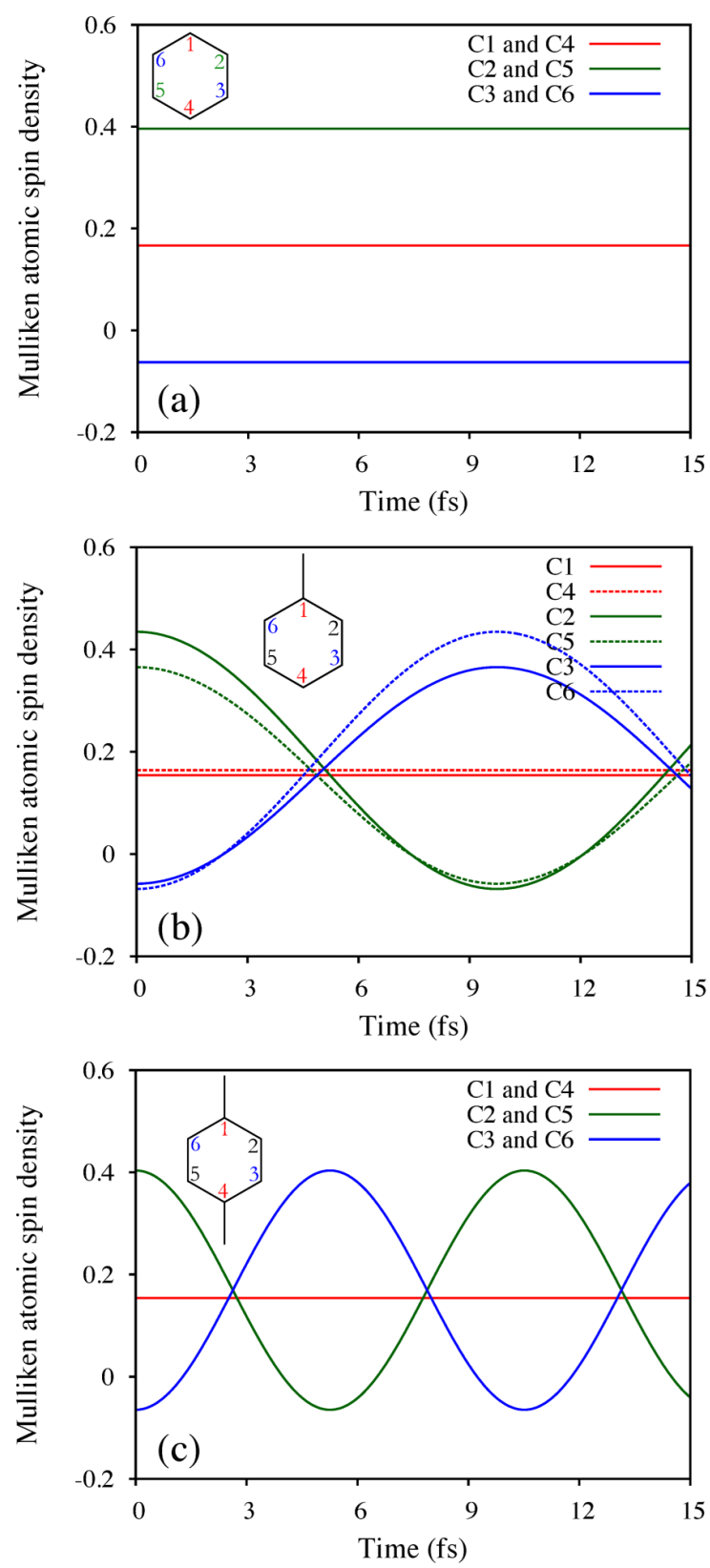

electron and the nuclear dynamics. Indeed, the structures can refer both to the nature of the electronic density and to the nuclear geometry.

To follow the evolution of the electronic wavefunction, its spin density-that allows one to locate the unpaired electron-is computed and partitioned onto the atoms at each step of the simulation. The evolution of the partitioned spin densities is shown in Figure 2. By assigning a different spin density pattern to each VB structure of the moat, one can then decompose the spin density of the time-dependent electronic wavefunction in the space of VB structures and plot the electron dynamics "trajectory" on the moat diagram (Figure 3) where the structures represent exclusively the nature of the electronic wavefunction in this case. Note that because each structure is a superposition of two resonance
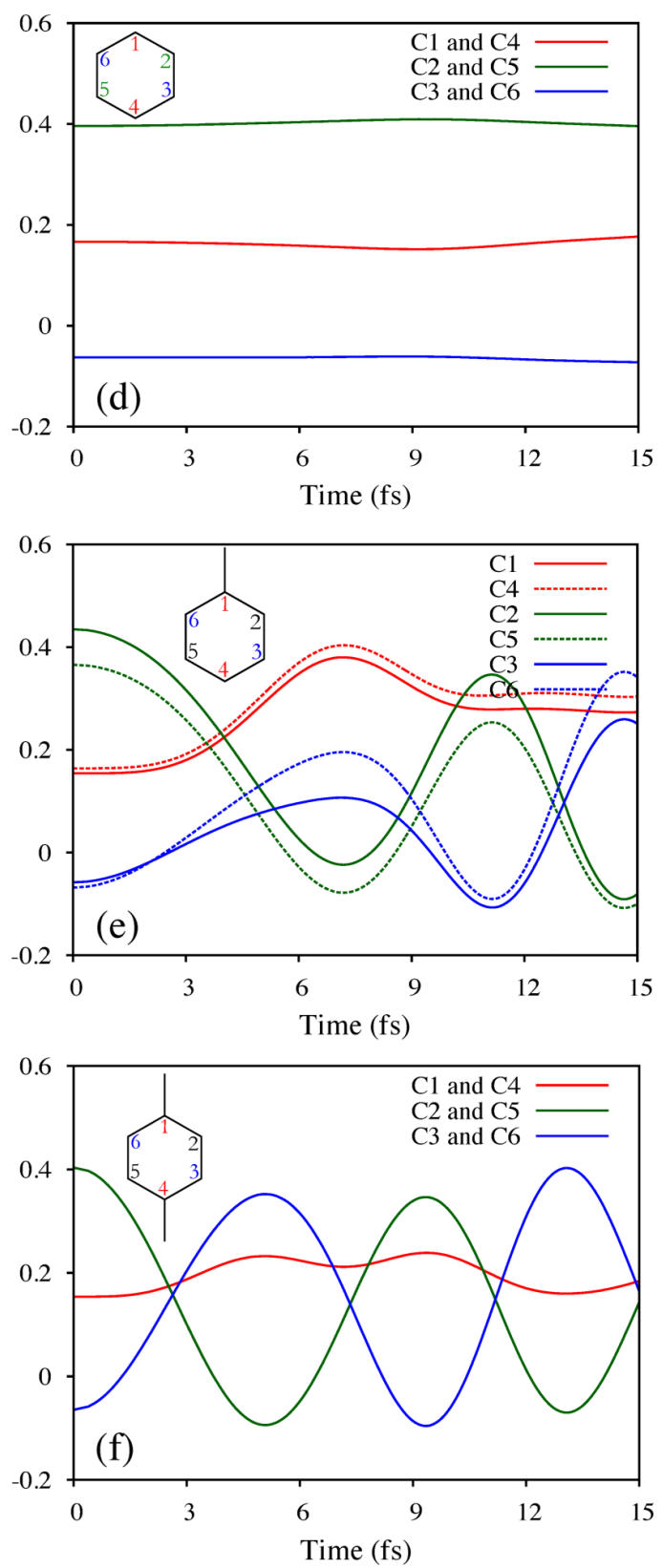

FIG. 2. Spin densities partitioned onto the atoms as a function of time after ionization of the three systems: benzene at the top (a) and (d), toluene in the middle (b) and (e), and para-xylene at the bottom (c) and (f). Simulations with fixed nuclei are on the left (a)-(c) and with nuclei moving on the right (d)-(f). The hexagons at the top left of each plot show the numbering of the carbon atoms: $\mathrm{C} 1$ and $\mathrm{C} 4$ in red, $\mathrm{C} 2$ and $\mathrm{C} 5$ in green, and $\mathrm{C} 3$ and $\mathrm{C} 5$ in blue. 

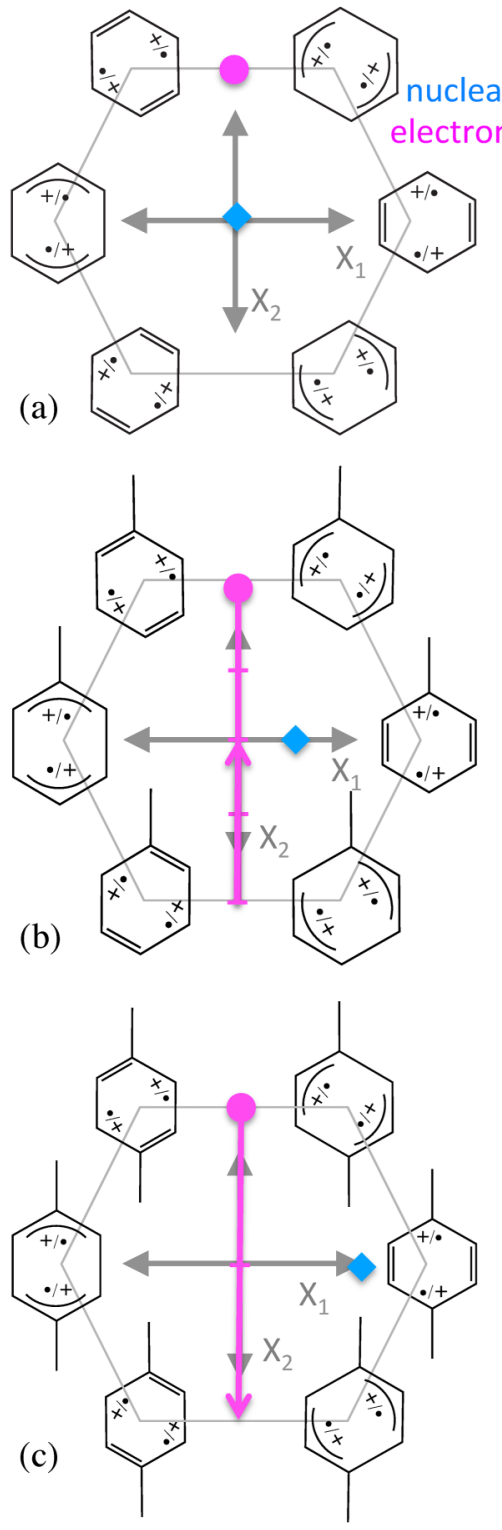

(e)

(d)
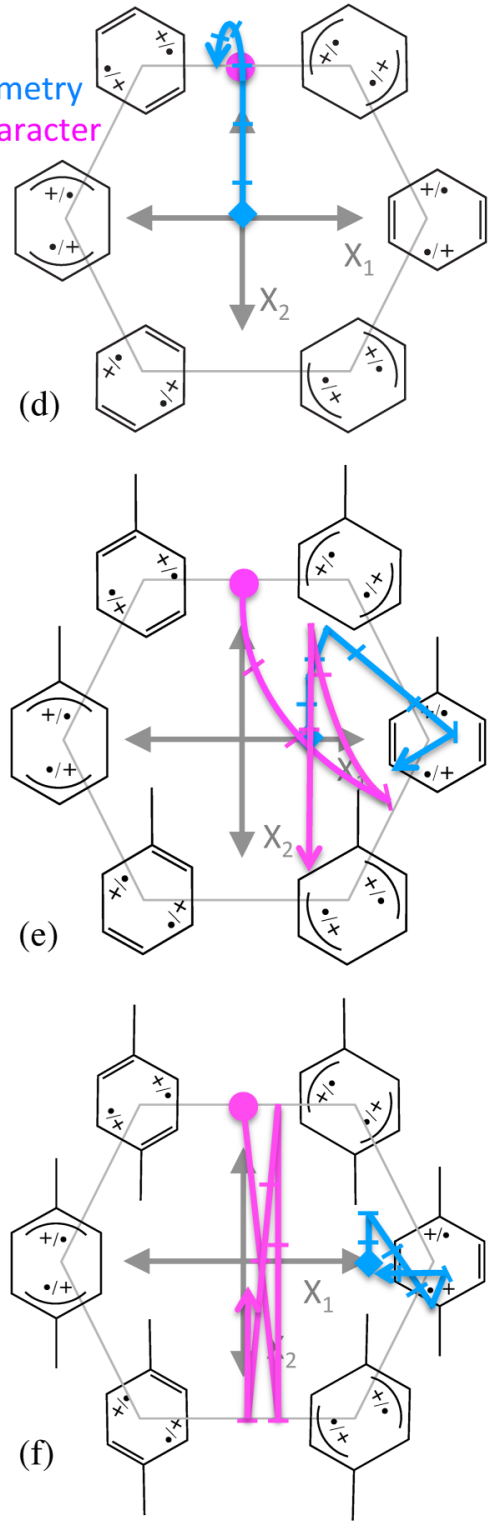

FIG. 3. Electron (in pink) and nuclear (in blue) dynamics after ionization represented in the structure diagram for the three systems: benzene at the top (a) and (d), toluene in the middle (b) and (e), and para-xylene at the bottom (c) and (f). Simulations with fixed nuclei are on the left (a)-(c) and with nuclei moving on the right (d)-(f). Time evolution is indicated with the presence of bars every $2.5 \mathrm{fs}$ on the trajectory arrows. Note that for each quinoid or antiquinoid structure, there are actually two resonance structures with the unpaired electron and the positive charge exchanged (as indicated by $\bullet /+$ ). structures where the unpaired electron and the positive charge are interchanged, locating the unpaired electron is equivalent to locating the positive charge. This is how we monitor the hole dynamics.

The nuclear trajectory can be plotted on the same moat diagram, but here, the structures represent the nuclear geometry exclusively (i.e., the pattern of single bonds corresponding to longer bond lengths versus double bonds corresponding to shorter bond lengths).

The evolution of the spin density in terms of VB structures, together with the nuclear trajectory in the branching space, is shown in Figure 3. By comparing them when both are represented in terms of VB structures, we can study how they differ. The electronic character and the nuclear geometry evolve either synchronously in equilibrium or asynchronously during the trajectory.

\section{Initial conditions}

For the three molecules studied, the initial nuclear geometry is taken as the equilibrium nuclear geometry of the neutral species (optimised at the B3LYP/6-31G* level of theory). It is represented by a blue diamond in Figure 3. In benzene cation, the initial nuclear geometry is at the crossing between $X_{1}$ and $X_{2}$, i.e., on the seam of CIs (Figures 3(a) and $3(\mathrm{~d}))$. It is displaced from the CI along $X_{1}$ in toluene cation (Figures 3(b) and 3(e)), and even further away from the CI along $X_{1}$ in para-xylene cation (Figures 3(c) and 3(f)). The simulations are started with no initial nuclear momentum. ${ }^{37}$

We are not aiming to reproduce a given experiment or to propose a specific experimental setup. Having said that, several electronic states can be populated coherently using for instance a pulse with a relatively large bandwidth. A coherent superposition of only the two lowest-energy eigenstates is reasonable in the three molecular systems studied here since at the equilibrium geometry of their neutral species, the two lowest-energy states are well separated in energy from the higher excited states: the energy gap between the ground and first excited states is small (see Table I) compared to the energy gap between the first and second excited states (more than $2 \mathrm{eV}$ ). In order to investigate the effects of chemical substitution and nuclear motion on electron dynamics, we 
TABLE I. Energy gap between the ground and first excited states of the cation at the equilibrium geometry of the neutral species (calculated at the CASSCF $(5,6) / 6-31 G^{*}$ level with the $6 \pi$ orbitals included in the active space) and corresponding expected period of oscillations (Eq. (14)).

\begin{tabular}{lcc}
\hline \hline Molecule & Energy gap $(\mathrm{eV})$ & Period of oscillations (fs) \\
\hline Benzene & 0.00 & $\infty$ \\
Toluene & 0.21 & 19.7 \\
Para-xylene & 0.39 & 10.6 \\
\hline \hline
\end{tabular}

choose as an initial electronic wavefunction for all three molecular systems, the equal mixture of the two lowest eigenstates in-phase: $\Psi(t=0)=\frac{1}{\sqrt{2}}\left(\psi_{0}+\psi_{1}\right)$. Note that even if only two states are initially populated, all eigenstates generated from the active space are included in the simulations. Because the methyl substituent groups perturb only slightly the electronic structure of benzene, the electronic character of the eigenstates is very similar for all three molecules and so is the electronic character of the initial superposition: high spin densities on atoms $\mathrm{C} 2$ and $\mathrm{C} 5$, medium densities on $\mathrm{C} 1$ and C4, and low densities on C3 and C6 (see Figure 2 for the initial partitioned spin densities and the numbering). ${ }^{38}$ In terms of $\mathrm{VB}$ structures, it corresponds to an electronic character intermediate between the two structures at the top (see pink dot in Figure 3).

\section{RESULTS AND DISCUSSION}

As outlined in the Introduction, the aim of the article is two-fold. First, we compare the electron dynamics results (simulated at fixed nuclear geometries) for the three different molecular systems to study the effect of chemical substitution on electron dynamics. Then, for each system, we compare the electron dynamics with fixed nuclei and with nuclei moving to study the effect of nuclear motion on electron dynamics.

The energy gaps between the ground and first excited states of the cations at the equilibrium geometries of the neutral species are given in Table I.

\section{A. Electron dynamics with fixed nuclei}

Let us first compare the electron dynamics of the three systems at fixed geometries to investigate the effect of chemical substitution. The results with fixed nuclei are shown on the left panels of Figures 2 and 3. Note that if the nuclei are not allowed to move, the populations of the two adiabatic states stay constant, i.e., 0.5 each.

\section{Benzene cation}

For benzene cation, there are no oscillations in the partitioned spin densities (Figure 2(a)) and there is no time evolution of the electronic character indicated in terms of VB structures (Figure 3(a)). Why is there no pure electron dynamics in this case? The electronic superposition is propagated with time but the phase factors of the eigenstates in Eq. (11) evolve at the same rate because the energies are degenerate. Therefore, the expected period of oscillationsinversely proportional to the energy gap-is infinite (see
Eq. (14) and Table I). Another way to explain the absence of pure electron dynamics is to realize that a superposition of two degenerate eigenstates is also an eigenstate. Therefore, the electronic wavefunction is stationary.

\section{Toluene cation}

For toluene cation, we now observe oscillations in the partitioned spin densities with a total period of about $20 \mathrm{fs}$ (Figure 2(b)). The latter can be directly related to the energy gap between the populated eigenstates at this geometry (Table I), using Eq. (14). Note that unlike benzene, the spin densities on opposite carbon atoms of the ring are not identical any more because of the asymmetry of the molecule. In Figure 3(b), we see oscillations between the top and the bottom VB structures. The bars along the pink arrow indicate time steps of $2.5 \mathrm{fs}$.

\section{Para-xylene cation}

For para-xylene cation, we observe oscillations this time with a total period of about 10 fs (Figure 2(c)), which again can be directly related to the energy gap between the populated eigenstates at this geometry (Table I) using Eq. (14). Note that the spin densities on opposite carbon atoms of the ring are again equal: the symmetry is recovered (partially) by adding a second methyl group in para position. In Figure 3(c), we see oscillations between the top and the bottom VB structures, as in toluene cation, but the spaces between the bars along the pink arrow are twice as long, indicating an oscillation in the electronic density that is twice as fast. This is due to a larger energy gap at the initial geometry that is further displaced away from the CI.

We observe that by adding methyl groups onto the benzene ring, the position of the equilibrium geometry of the neutral species with respect to the CI in the cation is changed: the nuclear geometry of the neutral species is exactly on the seam of CIs in benzene cation whereas it is displaced along $X_{1}$ in toluene cation and even further displaced along $X_{1}$ in para-xylene cation. The removal of degeneracy is necessary to observe any electron dynamics. Because the methyl substituents perturb only slightly the electronic structure of the benzene cation and because the equilibrium geometries of neutral toluene and para-xylene are both displaced from the CI along $X_{1}$, the electronic character of the two lowest adiabatic states at the initial geometry is similar in both systems: the ground electronic state has a quinoid character and the first excited state has an antiquinoid character. Therefore, the terms $\left|\psi_{0}(r ; R)\right|^{2},\left|\psi_{1}(r ; R)\right|^{2}$ and $\psi_{0}(r ; R) \psi_{1}(r ; R)$ in Eq. (13) are very similar for both toluene and para-xylene and, as a consequence, so are the nature of the created hole and its oscillating motion. The amplitude of the oscillations is the same because $\left|c_{0}\right|^{2}=\left|c_{1}\right|^{2}=0.5$ has been taken for both systems. The noticeable difference is the period of the oscillations, faster in para-xylene because of a larger energy gap between the ground and first excited states (see Eq. (14) and Table I). The results show how to use chemical substitution to control the timescale of electron dynamics keeping its nature unchanged. 


\section{B. Electron dynamics with nuclei moving}

In most theoretical studies of electron dynamics, the nuclei are kept fixed. We can test the validity of the fixednuclei approximation by comparing the simulations in Sec. IV A where the nuclear geometry was kept fixed with simulations where the nuclei are allowed to move. The results with nuclei moving are shown on the right panels of Figures 2 and 3. The time evolution of the ground and first excited state populations is shown in Figure 4.
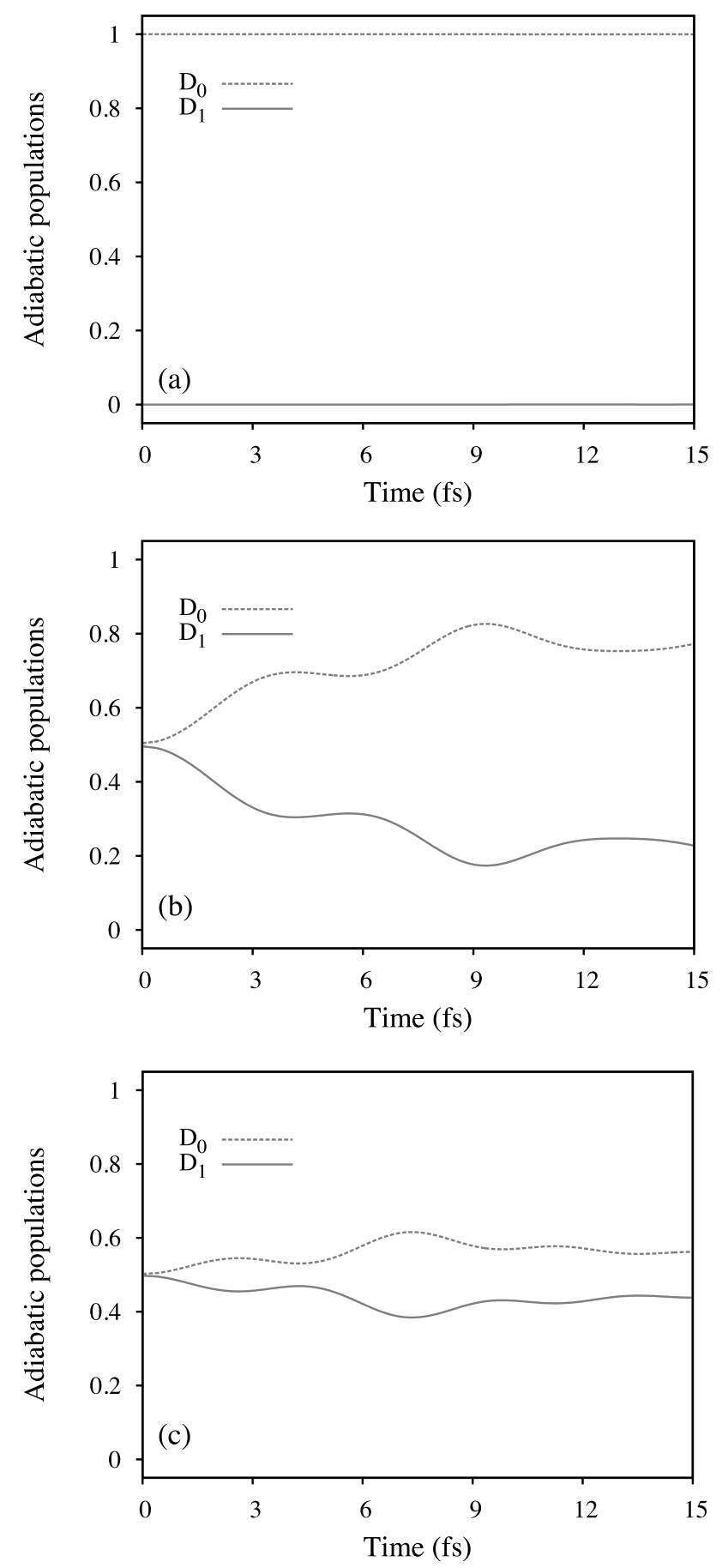

FIG. 4. Adiabatic populations of the ground and first excited states as a function of time after ionization of the three systems: benzene at the top (a), toluene in the middle (b), and para-xylene at the bottom (c). Note that in these simulations, nuclear motion is allowed (the adiabatic populations stay constant in the simulations with fixed nuclei).

\section{Benzene cation}

For benzene cation, we do not see a significant change in the electronic character during the first 15 fs (Figures 2(d) and 3(d)), similar to the case of fixed nuclear geometry. The nuclear geometry relaxes though (blue trajectory in Figure 3(d)). It adopts the geometry in equilibrium with the electronic character of the system: the blue arrow is superimposed on the pink dot. Note that the electronic wavefunction has totally decayed onto the ground state on an ultrafast timescale due to the infinitely large interstate coupling at the CI (see Figure 4(a)). On a longer timescale (several tens of fs), one expects synchronous electron and nuclear dynamics, also called charge transfer in the literature.

\section{Toluene cation}

For toluene cation, the evolution of the partitioned spin densities with nuclei moving (Figures 2(e) and 3(e)) is only identical to the one in the case of fixed nuclei (Figures 2(b) and 3(b)) up to 2-3 fs; they start to differ after then. There is still some kind of oscillating motion in the electronic density but not perfectly sinusoidal oscillations. First, the electron dynamics becomes faster: within $15 \mathrm{fs}, 0.75$ of an oscillation happens with fixed nuclei (Figure 3(b)) and 1.5 oscillations with nuclei moving (Figure 3(e)). Second, the nature of the oscillations is changed: the spin densities on atoms $\mathrm{C} 1$ and $\mathrm{C} 4$ are not constant any more (Figure 2(e)). Therefore, the symmetry of the electron dynamics is broken: the electronic "trajectory" in Figure 3(e) is displaced towards the right half of the diagram which represents the increase of the spin densities on $\mathrm{C} 1$ and $\mathrm{C} 4$. (In the quinoid structure on the far right of the diagram, the unpaired electron is localized on $\mathrm{C} 1$ and $\mathrm{C} 4$.)

These results show significant differences in the electron dynamics simulated with fixed nuclei and nuclei moving. This implies a strong interaction between the electronic and nuclear degrees of freedom. How can we explain the changes in both the nature and the timescale of the electron dynamics when the nuclear geometry is allowed to distort? The nuclear trajectory in the branching space is represented by the blue arrow in Figure 3(e): it moves along $X_{2}$ during the first 5 fs (to adopt a geometry in equilibrium with the initial electronic character $)^{26}$ and then acquires some component along $X_{1}$, moving away from the CI. Note that the nuclear and electronic trajectories are not superimposed within the first 15 fs: this means the nuclear and electron dynamics are asynchronous. Nuclear motion in the branching space results in a change of the electronic character of the adiabatic states, i.e., a change in the terms $\left|\psi_{0}(r ; R)\right|^{2},\left|\psi_{1}(r ; R)\right|^{2}$, and $\psi_{0}(r ; R) \psi_{1}(r ; R)$ in Eq. (13). In addition, the nuclear motion leads to a transfer of population between the two states through the non-adiabatic coupling. We observe a partial decay of the electronic wavefunction onto the ground state (Figure 4(b)): at $t=15 \mathrm{fs}$, the weight of the ground state is about 0.8 compared to 0.5 initially. As a result, the nature of the oscillations of the electronic density changes. In addition, the nuclear trajectory moves away from the CI, towards geometries where the energy gap between ground and first excited states is larger: this results in a speeding up of the oscillations (see Eq. (14)). The results show how the nuclear 
motion affects both the nature of the oscillating motion in the electronic density and its timescale after only 2-3 fs.

\section{Para-xylene cation}

For para-xylene cation, the evolution of the partitioned spin densities with nuclei moving (Figures 2(f) and 3(f)) is again very similar to the one in the case of fixed nuclei (Figures 2(c) and 3(c)) up to 2-3 fs. After then, we observe the same differences as in toluene cation (but less pronounced). The electron dynamics becomes faster: within $15 \mathrm{fs}, 1.5$ oscillations happen with fixed nuclei (Figure 3(c)) and 1.75 oscillations with nuclei moving (Figure 3(f)). The symmetry is also broken with the variation of the spin densities on atoms $\mathrm{C} 1$ and $\mathrm{C} 4$ (Figure 2(f)) and a displacement of the electronic "trajectory" towards the right half of the diagram in Figure 3(f).

Similar arguments to those used for toluene cation can explain the differences in the electron dynamics with fixed and moving nuclei. Yet, the differences are much less pronounced in para-xylene cation than in toluene cation. Why do the electronic and nuclear degrees of freedom seem to interact less? The nuclear geometry follows the potential energy gradient towards a geometry in equilibrium with the electronic character. In benzene cation, the electronic character is stationary and the nuclear trajectory reaches the equilibrium geometry within the first $10 \mathrm{fs}$. In toluene and para-xylene cations, the electronic character evolves with time and so does the nuclear gradient: an electronic character on the opposite side of the diagram leads to a nuclear gradient in the opposite direction in the branching space. The electronic character changing faster in para-xylene cation than in toluene cation, the nuclear gradient reverses faster, leading to less nuclear motion. This may also be amplified by a derivative coupling of smaller magnitude in para-xylene cation (geometry further away from the CI). ${ }^{26}$ The combination of a slower nuclear motion and a smaller non-adiabatic coupling leads to almost no decay of the electronic wavefunction onto the ground state: at $15 \mathrm{fs}$, the weight of the ground state is still about 0.55 (Figure 4(c)). These reasons may explain (i) a smaller change in the two first terms and $\psi_{0}(r ; R) \psi_{1}(r ; R)$ in the third term of Eq. (13) and, as a consequence, a smaller change in the nature of the oscillations and (ii) a smaller change in the energy gap between ground and first excited states and, as a consequence, a smaller change in the period of the oscillations using Eq. (14). The nuclear motion seems therefore to have less effect on the electron dynamics in para-xylene cation.

\section{CONCLUSION}

In the present article, we have studied the electron dynamics following the outer valence ionization in three different but related molecules: benzene and two methylsubstituted benzenes, toluene and para-xylene. The methyl substitution does not alter the electronic structure significantly but breaks the 6-fold symmetry: the equilibrium geometry of the neutral species that corresponds to a $\mathrm{CI}$ in the cation of benzene molecule is now displaced away from the CI in toluene and para-xylene cations. As a result, the removal of degeneracy creates a non-stationary state that leads to electron dynamics in the methyl-substituted benzenes while no pure electron dynamics is observed in benzene. Also, a larger energy gap between the populated ground and first excited states in para-xylene cation produces faster electron dynamics. So the effect of the chemical substitution on the electron dynamics is important: the result changes from no electron dynamics at all in benzene cation to some electron dynamics in toluene cation and finally even faster electron dynamics in para-xylene cation. It shows how one can use chemical substitution to control for instance the timescale of electron dynamics keeping its nature unchanged.

We have compared pure electron dynamics simulations with simulations of coupled electron-nuclear dynamics using the Ehrenfest approximation. In toluene cation, only 2-3 fs are necessary for the nuclear geometry to distort enough to affect the electron dynamics (within the Ehrenfest approximation). This time may seem rather short considering the nuclei move on a fs timescale. But near a conical intersection, the interstate coupling is large and small nuclear displacements lead to large changes in the electronic character of the adiabatic states. The nature of the oscillations is changed because the system moves to geometries where the definition of the adiabatic states changes and/or because the adiabatic populations change. Also, the period of the oscillations is affected by the evolution of the energy gap between the two eigenstates populated. In para-xylene cation, the initial electron dynamics seems to be too fast for the nuclear motion to have a very significant effect, as was the case for other systems we have studied. ${ }^{15,17}$

\section{ACKNOWLEDGMENTS}

This work was supported by UK-EPSRC Grant No. EP/I032517/1. All calculations were run using the Imperial College High Performance Computing service. The authors thank Felicity McGrath and Jon P. Marangos for helpful discussions.

${ }^{1}$ J. Breidbach and L. S. Cederbaum, J. Chem. Phys. 118, 3983 (2003).

${ }^{2}$ A. I. Kuleff and L. S. Cederbaum, J. Phys. B: At., Mol. Opt. Phys. 47, 124002 (2014)

${ }^{3}$ M. F. Kling and M. J. J. Vrakking, Annu. Rev. Phys. Chem. 59, 463 (2008).

${ }^{4}$ F. Krausz and M. Y. Ivanov, Rev. Mod. Phys. 81, 163 (2009).

${ }^{5}$ L. Belshaw, F. Calegari, M. J. Duffy, A. Trabattoni, L. Poletto, M. Nisoli, and J. B. Greenwood, J. Phys. Chem. Lett. 3, 3751 (2012).

${ }^{6}$ F. Lepine, M. Y. Ivanov, and M. J. J. Vrakking, Nat. Photonics 8, 195 (2014).

${ }^{7}$ F. Calegari, D. Ayuso, A. Trabattoni, L. Belshaw, S. D. Camillis, S. Anumula, F. Frassetto, L. Poletto, A. Palacios, P. Decleva, J. B. Greenwood, F. Martín, and M. Nisoli, Science 346, 336 (2014).

${ }^{8}$ F. Remacle and R. D. Levine, Z. Phys. Chem. 221, 647 (2007).

${ }^{9}$ A. I. Kuleff and L. S. Cederbaum, Chem. Phys. 338, 320 (2007).

${ }^{10}$ S. Lünnemann, A. I. Kuleff, and L. S. Cederbaum, Chem. Phys. Lett. 450, 232 (2008).

${ }^{11}$ S. Lünnemann, A. I. Kuleff, and L. S. Cederbaum, J. Chem. Phys. 129, 104305 (2008)

${ }^{12}$ G. Periyasamy, R. D. Levine, and F. Remacle, Chem. Phys. 366, 129 (2009).

${ }^{13}$ A. I. Kuleff, S. Lünnemann, and L. S. Cederbaum, J. Phys. Chem. A 114, 8676 (2010).

${ }^{14}$ I. S. Ulusoy and M. Nest, J. Phys. Chem. A 116, 11107 (2012).

${ }^{15}$ D. Mendive-Tapia, M. Vacher, M. J. Bearpark, and M. A. Robb, J. Chem. Phys. 139, 044110 (2013).

${ }^{16}$ M. Vacher, D. Mendive-Tapia, M. J. Bearpark, and M. A. Robb, Theor. Chem. Acc. 133, 1505 (2014). 
${ }^{17}$ M. Vacher, M. J. Bearpark, and M. A. Robb, J. Chem. Phys. 140, 201102 (2014).

${ }^{18}$ B. E. Applegate, T. A. Barckholtz, and T. A. Miller, Chem. Soc. Rev. 32, 38 (2003).

${ }^{19}$ M. J. Paterson, M. J. Bearpark, M. A. Robb, L. Blancafort, and G. A. Worth, Phys. Chem. Chem. Phys. 7, 2100 (2005).

${ }^{20}$ K. F. Hall, A. M. Tokmachev, M. J. Bearpark, M. Boggio-Pasqua, and M. A. Robb, J. Chem. Phys. 127, 134111 (2007).

${ }^{21}$ W. Domcke and D. R. Yarkony, Annu. Rev. Phys. Chem. 63, 325 (2012).

${ }^{22}$ M. A. Robb, "Conical intersections in organic photochemistry," in Conical Intersections: Theory, Computation and Experiment, edited by W. Domcke, D. R. Yarkony, and H. Köppel (World Scientific Publishing, 2011), Chap. 1, pp. 3-50.

${ }^{23}$ D. R. Yarkony, Rev. Mod. Phys. 68, 985 (1996).

${ }^{24}$ A. Stolow, Faraday Discuss. 163, 9 (2013).

${ }^{25}$ Another way to think about the limitation of the fixed-nuclei approximation is to realize that the non-adiabatic coupling is inversely proportional to the energy gap. Therefore, as the energy gap decreases, the non-adiabatic coupling increases and the interaction between the electronic and nuclear degrees of freedom is particularly strong. Near conical intersections, the Born-Oppenheimer approximation breaks down and one cannot think in terms of the electronic density adapting instantaneously to a fixed nuclear geometry.

${ }^{26}$ M. Vacher, J. Meisner, D. Mendive-Tapia, M. J. Bearpark, and M. A. Robb, "Electronic control of initial nuclear dynamics adjacent to a conical intersection," J. Phys. Chem. A (published online 2014).

${ }^{27}$ J. M. Millam, V. Bakken, W. Chen, W. L. Hase, and H. B. Schlegel, J. Chem. Phys. 111, 3800 (1999).

${ }^{28}$ M. J. Frisch, G. W. Trucks, H. B. Schlegel, G. E. Scuseria, M. A. Robb, J. R. Cheeseman, G. Scalmani, V. Barone, B. Mennucci, G. A. Petersson, H. Nakatsuji, M. Caricato, X. Li, H. P. Hratchian, A. F. Izmaylov, J. Bloino, G. Zheng, J. L. Sonnenberg, M. Hada, M. Ehara, K. Toyota, R. Fukuda, J. Hasegawa, M. Ishida, T. Nakajima, Y. Honda, O. Kitao, H. Nakai, T. Vreven, J. A. Montgomery, Jr., J. E. Peralta, F. Ogliaro, M. Bearpark, J. J. Heyd,
E. Brothers, K. N. Kudin, V. N. Staroverov, R. Kobayashi, J. Normand, K. Raghavachari, A. Rendell, J. C. Burant, S. S. Iyengar, J. Tomasi, M. Cossi, N. Rega, J. M. Millam, M. Klene, J. E. Knox, J. B. Cross, V. Bakken, C. Adamo, J. Jaramillo, R. Gomperts, R. E. Stratmann, O. Yazyev, A. J. Austin, R. Cammi, C. Pomelli, J. W. Ochterski, R. L. Martin, K. Morokuma, V. G. Zakrzewski, G. A. Voth, P. Salvador, J. J. Dannenberg, S. Dapprich, A. D. Daniels, Ö. Farkas, J. B. Foresman, J. V. Ortiz, J. Cioslowski, and D. J. Fox, Gaussian Development Version, Revision H.10, Gaussian, Inc., Wallingford, CT, 2010.

${ }^{29}$ J. Almlöf and P. R. Taylor, Int. J. Quantum Chem. 27, 743 (1985).

${ }^{30}$ G. Hunter, Int. J. Quantum Chem. 9, 237 (1975).

${ }^{31}$ A. Abedi, N. T. Maitra, and E. K. U. Gross, Phys. Rev. Lett. 105, 123002 (2010).

${ }^{32}$ L. S. Cederbaum, J. Chem. Phys. 138, 224110 (2013).

${ }^{33}$ E. J. Heller, J. Chem. Phys. 62, 1544 (1975).

${ }^{34}$ D. V. Makhov, W. J. Glover, T. J. Martinez, and D. V. Shalashilin, J. Chem. Phys. 141, 054110 (2014).

${ }^{35}$ G. A. Worth, M. A. Robb, and B. Lasorne, Mol. Phys. 106, 2077 (2008).

${ }^{36}$ B. O. Roos, P. R. Taylor, and P. E. M. Siegbahn, Chem. Phys. 48, 157 (1980).

${ }^{37}$ Our previous study ${ }^{26}$ of nuclear dynamics in toluene cation shows that the average of an ensemble of trajectories-generated by geometry sampling to take into account nuclear zero-point energy of the neutral species-is similar to a single un-sampled trajectory started at the equilibrium geometry with no initial kinetic energy. Since here the focus is electron dynamics, we use this single nuclear trajectory for each system to study how electron dynamics changes with chemical substitution and after nuclear motion.

${ }^{38}$ The asymmetry between the atoms $\mathrm{C} 2 / \mathrm{C} 5$ and $\mathrm{C} 3 / \mathrm{C} 6$ is due to the interference term in the electronic density (Eq. (13)). The electronic superposition $\frac{1}{\sqrt{2}}\left(\psi_{0}+\psi_{1}\right)$ leads to higher spin densities on C2 and C5 than on C3 and C6. The superposition $\frac{1}{\sqrt{2}}\left(\psi_{0}-\psi_{1}\right)$ would lead to opposite spin densities, i.e., higher spin densities on $\mathrm{C} 3$ and $\mathrm{C} 6$ than on $\mathrm{C} 2$ and $\mathrm{C} 5$. The latter superposition can be seen as a phase shift of $180^{\circ}$ (reached at $t=T / 2$ in simulations with fixed nuclei). 